[Radiocarbon, Vol. 11, No. 2, 1969, P. 502-508]

\title{
TATA INSTITUTE RADIOCARBON DATE LIST VII
}

\section{P. AGRAWAL, S. K. GUPTA, and SHEELA KUSUMGAR}

Tata Institute of Fundamental Research, Bombay 5, India

We continue to give dates based on the half-life of 5568 years according to the decision of the Sixth Pullman Conference (Internatl. Conf., Pullman, 1965). The year 1950 has been used as a reference year for converting the dates to A.D./B.C. scale. A value corresponding to $95 \%$ net counting rate of the NBS oxalic acid has been used as the modern reference standard.

To obtain more dates for the Late Quaternary, we have dated samples from Kakathope (TF-695, -696), Malia (TF-807(a), -807(b)), and Sambhar Lake (TF-698, -738, -739), using $\mathrm{C}^{14}$ dates based on $\tau_{1 / 2}=$ $5730 \mathrm{yr}$. With the help of the pollen sequence, worked out by the Sahni Institute of Palaeobotany, Lucknow, and $\mathrm{C}^{14}$ dates for the different pollen zones, an absolute chronology for the Late Quaternary of India is being built up.

A few of the $\mathrm{C}^{14}$ dates from the earlier Kayatha excavations had shown some scatter. Therefore more samples were collected from fresh excavations conducted by the Deccan College, Poona. Dates obtained are consistent and place the beginning of the Kayatha culture (TF-776 to TF-781) to ca. 1900 в.c.

The date (TF-748) for Kodekal again shows that the southern neolithic cultures show increasingly younger dates to the south (Radiocarbon, 1968, v. 10, p. 13).

The enigmatic Pirak ware was assigned a very high antiquity by Raikes (1963), but TF-861 places it at ca. 800 B.C. in agreement with Casal's estimates (pers. commun.).

\section{ACKNOWLEDGMENT}

We thank Prof. D. Lal for his guidance. Mr. S. V. Kerkar helped us in laboratory work. We are also thankful to Drs. H. D. Sankalia, Vishnu Mittre, and Gurdip Singh for cooperation in our Late Quaternary dating program.

\section{SAMPLE DESCRIPTIONS}

ARCHAEOLOGIC SAMPLES

\section{A. India}

TF-576. Hallur, India, Neolithic culture

Charcoal from Hallur ( $14^{\circ} 20^{\prime} \mathrm{N}$ Lat, $75^{\circ} 37^{\prime} \mathrm{E}$ Long), Dist. Dharwar, Trench 1, Layer 8, depth $3.6 \mathrm{~m}$, Field No. HLR 1965. Subm. by M. S. Nagaraja Rao, Kannada Research Inst., Karnatak Univ., Dharwar.

\section{Kayatha series, Madhya Pradesh}

Kayatha $\left(23^{\circ} 14^{\prime} \mathrm{N}\right.$ Lat, $76^{\circ} 02^{\prime} \mathrm{E}$ Long), Dist. Ujjain is Chalcolithic site. Samples subm. by H. D. Sankalia, Deccan College, Poona-6. Except for TF-780, all samples were given $\mathrm{NaOH}$ pretreatment. 


\section{TF-776. Chalcolithic culture}

Charcoal, Trench KTH-A, Layer 4, Field No. 111.

TF-777. Chalcolithic culture

Charcoal, Trench KTH-A, Layer 5, Field No. 361.

TF-778. Chalcolithic culture

Charcoal, Trench KTH-A, Layer 6, Field No. 599.

TF-779. Chalcolithic culture

Charcoal, Trench KTH-A, Layer 7, Field No. 693.

TF-780. Chalcolithic culture

Charcoal, Trench KTH-A, Layer 8, Field No. 697.

TF-781. Chalcolithic culture

Charcoal, Trench KTH-A, Layer 9, Field No. 976.

TF-748. Kodekal, India, Neolithic culture

Charcoal from Kodekal ( $16^{\circ} 21^{\prime} \mathrm{N}$ Lat, $76^{\circ} 24^{\prime}$ E Long), Dist. Gulbarga, Trench 2, Layer 4, depth 2 to $3 \mathrm{~m}$, Field No. 948. Subm. by H. D. Sankalia.

\section{Moosakhand Dam series, Uttar Pradesh}

Iron tools and pottery from Moosakhand Dam $\left(24^{\circ} 59^{\prime} \mathrm{N}\right.$ Lat, $83^{\circ}$ $18^{\prime}$ E Long), Dist. Varanasi, from pits left exposed 2 to 3 months before. Subm. by V. S. Krishnaswamy, Geol. Survey, India, E. G. Div., N Region, Lucknow. All samples given $\mathrm{NaOH}$ pretreatment.

\section{TF-819(c). River bank deposits}

A.D. 1610

$340 \pm 95$

Carbonized wood, depth $15.22 \mathrm{~m}$. Field No. MKD/lc.

TF.820. River bank deposits

Modern $\mathrm{MKD} / 2$.

Wood with leaves and twigs in sand silt, depth $15.22 \mathrm{~m}$, Field No.

TF-821. River bank deposits

Modern

Wood, depth $3 \mathrm{~m}$, Field No. MKD/3.

TF-701. Palvoy, India, Neolithic culture

$3805 \pm 100$

1855 B.C.

Charcoal from Palvoy (14 $31^{\prime} \mathrm{N}$ Lat, $77^{\circ} 09^{\prime} \mathrm{E}$ Long), Dist. Ananthpur, Layer 9, depth 3.5 m. Subm. by H. D. Sankalia. Comment: $\mathrm{NaOH}$ pretreatment. 
TF-636. Rajbadidanga, India, historical

$1665 \pm 95$

Charcoal from Rajbadiclanga (24 $1^{\prime} 44^{\prime \prime} \mathrm{N}$ Lat, $88^{\circ} 11^{\prime} 04^{\prime \prime}$ E Long), Dist. Murshidabad, Trench B-5, Layer 8 , depth $3.3 \mathrm{~m}$. Subm. by S. R. Das, Dept. Archaeol., Univ. Calcutta.

\section{Takalghat series, Maharashtra}

Takalghat, Dist. Nagpur, excavated by S. B. Deo, Nagpur Univ., Nagpur, who subm. samples. Similar painted pottery was also found at Paunar where it could be assigned to earliest pre-iron occupation ca. 600 to 800 B.C.

\section{TF-783. Paunar I complex}

$2495 \pm 100$

Charcoal, Trench TKG-I, $1.95 \times 1.60 \mathrm{~m}$, Layer $9(\mathrm{~A})$, depth 4.78 m, Field No. TKG-215.

\section{TF-784. Paunar I complex}

Charcoal, Trench TKG-I, Locus III' $\times$ IV' $^{\prime} .75 \times 125 \mathrm{~m}$, Layer $7(\mathrm{~A})$, depth 3.03 m. Field No. TKG-216.

\section{Ter series, Maharashtra}

Ter, Dist. Osmanabad, excavated by M. G. Dikshit, Dir. Archaeol., Maharashtra, Bombay, who subm. samples. $\mathrm{NaOH}$ pretreatment given all samples.

\section{TF-746. Historical}

$1645 \pm 100$

Charcoal lrom Layer 3, Reg. No. 5/619.

A.D. 305

\section{TF-747. Historical}

$2045 \pm 100$

Charcoal irom Layer 22, Reg. No. 5/573.

95 в.c.

TF-749. Wood figure

Modern

Wooden Ganesa figure dated to determine authenticity as antique object. Subm. by M. G. Dikshit.

\section{B. Poland}

\section{Nieborowa series, Poland}

Nieborawa, Dist. Chelm Lubelski, Poland, excavated by Chmielenski, Warsaw 22, St. Niemcewicza 24-45. $\mathrm{NaOH}$ pretreatment given both samples.

\section{TF-754. Neolithic culture}

Charcoal from fireplace, Locus CCXL-CCXLI-023, Layer 3, depth $0.55 \mathrm{~m}$. Field No. NBI/5/C-67-1. Comment: sample expected to date end of Neolithic period. 
TF-755. Unknown culture

Charcoal from fireplace, Locus CCXXXIV-014 and 015, Layers 3 and 4, depth $0.48 \mathrm{~m}$, Field No. NBI/5/C-67-2.

\section{Pakistan}

\section{TF-861. Pirak, W Pakistan}

$2660 \pm 100$

Charcoal from Pirak $\left(29^{\circ} 30^{\prime} \mathrm{N}\right.$ Lat, $67^{\circ} 54^{\prime}$ E Long), Dist. Kachi, Layer W 12, depth 1 m, Field No. Pk. A. Coll. and subm. by J. M. Catsal, Mus. Guimet, Paris.

$$
\text { I. Nepal }
$$

$2235 \pm 95$

TF-737. Tilaurakot, Nepal, P.G. ware deposits

285 B.C.

Charcoal from Tilaurakot $\left(27^{\circ} 34^{\prime} 30^{\prime \prime} \mathrm{N}\right.$ Lat, $83^{\circ} 30^{\prime} 3^{\prime \prime} \mathrm{E}$ Long) Dist. Taulihawa, Locus A2-A3, $4.9 \times 1 \mathrm{~m}$, Layer 10 , depth $2.58 \mathrm{~m}$, Field No. TLK-6. Subm. by N. R. Banerjee, Dept. Archaeol. Govt. of Nepal, Kathmandu.

\section{E. Thailand}

\section{TF-802. Spirit Cave, Thailand, Mesolithic}

$8505 \pm 135$

Wood from Spirit Cave $\left(20^{\circ} \mathrm{N}\right.$ Lat, $98^{\circ} \mathrm{E}$ Long), Mae Hongson Prov. Locus B2-B3, Layer 2A, depth 0.22 m, Field No. B2-B3 (2A). Subm. by C. F. Gorman, Archaeol. Lab., Univ. of Hawaii, Honolulu.

\section{LATE QUATFRNARY SAMPLES}

\section{Kakathope series, Madras}

Kakathope $\left(11^{\circ} 35^{\prime} \mathrm{N}\right.$ Lat, $70^{\circ} 52^{\prime} \mathrm{E}$ Long), Dist. Nilgiris. Samples recovered by boring for dating pollen zones. Subm. by Vishnu-Mittre, Birbal Sahni Inst. of Paleobotany, Lucknow. $\mathrm{NaOH}$ pretreatment was given to all samples.

\section{TF-695. Late Pleistocene}

$$
\begin{aligned}
& 14,980+355 \\
&-\mathbf{3 4 0} \\
& 13,030 \text { в.C. }
\end{aligned}
$$

Organic mud, depth 2.0 to $2.30 \mathrm{~m}$, Field No. $4789 / 3$.

$$
\begin{aligned}
23,590 & +740 \\
& -675 \\
21,640 & \text { в.C. }
\end{aligned}
$$

TF-696. Late Pleistocene

Organic mud, depth 3.20 to $3.50 \mathrm{~m}$, Field No. $4789 / 3$.

\section{Malia series, Gujarat}

Malia (23 $05^{\prime} 30^{\prime \prime} \mathrm{N}$ Lat, $70^{\circ} 45^{\prime} 30^{\prime \prime} \mathrm{E}$ Long) in Little Rann of Kutch excavated by Indian Railways. Samples obtained from boreholes drilled for construction of railway embankment. Subm. by Y. G. K. Murty, Dir., Gujarat Circle, Geol. Survey India, Ahmedabad. 


\section{TF-807(a). Late Pleistocene}

$13,640 \pm 200$

Shells from Borehole 6 , at m thick. Field No. 7.

\section{TF-807(b). Late Pleistocene}

$15,995 \pm 250$

Shells from Borehole 6, depth $19.85 \mathrm{~m}$, bottom of bed. Field No. 7.

\section{TF.808. Holocene}

Shells from creek cutting between Boreholes 9 and 10. Shells from depth $1.0 \mathrm{~m}$ and $1.3 \mathrm{~m}$ were mixed. Field Nos. $10 \mathrm{~A}$ and $10 \mathrm{~B}$.

\section{TF-837(b). Pleistocene}

Oyster shells from Khadir I. (23 $52^{\prime} 30^{\prime \prime} \mathrm{N}$ Lat, $70^{\circ} 27^{\prime} 30^{\prime \prime}$ E Long) in Great Rann of Kutch. Oyster debris represent raised beaches of subrecent times. Ca. $+3 \mathrm{~m}$. Subm. by S. K. Biswas, Oil and Natural Gas Commission, Baroda-4.

\section{Mangalore series, Mysore}

Mangalore $\left(12^{\circ} 56^{\prime} \mathrm{N}\right.$ Lat, $74^{\circ} 50^{\prime}$ E Long), Dist. S Kanara. Samples from boreholes on sea bottom. Subm. by H. N. Siddiqui, Marine Geol. Unit, Geol. Survey India, Calcutta.

\section{TF-740(b). Holocene}

$1985 \pm 90$

Shells from Borehole 59, depths 11.89 to $13.47 \mathrm{~m}$.

\section{TF-740(d). Holocene}

Carbonaceous clay from Borehole 59, depth 58.75 to $60.42 \mathrm{~m}$.

\section{TF-741. Holocene}

Shells from Borehole 64, depth 12.77 to $17.25 \mathrm{~m}$.

\section{TF-742. Holocene}

Shells from Borehole 69, depth 25.70 to $30.58 \mathrm{~m}$.

$$
1980 \pm 100
$$

30 B.C.

$$
1390 \pm 115
$$

\section{Sambhar Lake series, Rajasthan}

Sambhar salt lake (26 $54^{\prime} \mathrm{N}$ Lat, $75^{\circ} 13^{\prime} \mathrm{E}$ Long), Dist. Jaipur, excavated by G. Singh, Birbal Sahni Inst. of Paleobotany for pollen analysis and $\mathrm{C}^{14}$ dating. All samples given $\mathrm{NaOH}$ pretreatment.

\section{TF-698. Late Quaternary}

$8585 \pm 140$

Organic debris, depth 3.12 to 3.20 m, Field No. S-2/312-320, Sample No. RG-2. 
TF-738. Late Quaternary

$8065 \pm 135$

Organic debris, depth 2.70 to 2.85 m, Field No. S-2/270-285, Sample No. RC-3.

$4535 \pm 110$

TF-739. Late Quaternary

2585 в.c.

Organic debris, depth 1.50 to $1.60 \mathrm{~m}$, Field No. S-2/150-160, Sample No. RC-4.

III. GEOCHEMICAL SAMPLES

The following samples were collected by members of our Geophysics Group in connection with the study of problems relating to ground water dating. Interpretation of the data will be published elsewhere. Results are given as "real" ages.

TF-609. Palana, Rajasthan, open well

$4845 \pm 110$

Dissolved carbonates, picked up in IR-45 and IRA-400 anion exchange resins from open well at Palana ( $28^{\circ} \mathrm{N}$ Lat, $72^{\circ} 45^{\prime} \mathrm{E}$ Long), Dist. Bikaner. Sample coll. and subm. by V. N. Nizampurkar.

TF-456. Vijapur, Gujarat, tube well

$2215 \pm 120$

265 B.C.

Dissolved carbonates, picked up in IR-45 and IRA-400 anion exchange resins from tube well at Vijapur $\left(23^{\circ} 33^{\prime} \mathrm{N}\right.$ Lat, $72^{\circ} 50^{\prime} 38^{\prime \prime} \mathrm{E}$ Long), Dist. Mehsana. Sample coll. by V. N. Nizampurkar.

TF-686. Amritsar, Panjab, tube well

$2710 \pm 100$

760 B.C.

Dissolved carbonates, picked up in ASR-76 and IRA-400 anion exchange resins, from tube well at Amritsar $\left(29^{\circ} 45^{\prime} \mathrm{N}\right.$ Lat, $73^{\circ} 30^{\prime} \mathrm{E}$ Long), Dist. Amritsar. Sample coll. and subm. by V. N. Nizampurkar.

TF-687. Rupar, Panjab, tube well

$295 \pm 90$

Dissolved carbonates, picked up in IR-45 and IRA-400 anion exchange resins, from tube well at Rupar ( $31^{\circ} \mathrm{N}$ Lat, $76^{\circ} 40^{\prime} \mathrm{E}$ Long), Dist. Rupar. Sample coll. and subm. by V. N. Nizampurkar.

TF-688. Neyveli, Madras, tube well

$525 \pm 100$

Dissolved carbonates picked up in IR-45 and IRA-400 anion exchange resin from tube well at Neyveli ( $11^{\circ} 32^{\prime} \mathrm{N}$ Lat, $79^{\circ} 28^{\prime} \mathrm{E}$ Long), Dist. S Arcot. Sample coll. and subm. by V. N. Nizampurkar.

\section{TF-762. Laboratory tap}

Free and fixed $\mathrm{CO}_{2}$ from Lab. Tap water. Coll. 1967 and subm. by S. K. Gupta.
(a) Free $\mathrm{CO}_{2}$
$150.34 \pm 1.07 \%$ Modern
(b) Fixed $\mathrm{CO}_{2}$
$154.66 \pm 1.06 \%$ Modern 


\section{TF-763. Mulund, Maharashtra, tube well}

Free and fixed $\mathrm{CO}_{2}$ from tube well at Mulund $\left(19^{\circ} 05^{\prime} \mathrm{N}\right.$ Lat, $72^{\circ}$ $50^{\prime}$ E Long), Bombay. Coll. 1967 and subm. by S. K. Gupta.
(a) Free $\mathrm{CO}$.
$110.57 \pm 1.12 \%$ Modern
(b) Fixed $\mathrm{CO}_{2}$
$112.78 \pm 1.18 \%$ Modern

\section{Neyveli series, Madras}

Neyveli lignite field is in coastal plain of SE India. Study area is almost wholly overlain by sandstones, grits, clay beds, assoc. lignite beds, and water bearing sands of Neyveli artesian aquifer.

Samples were coll. from lignite mine area to study flow of ground water. Although Samples TF-811, -813, -815, -817 were roughly equidistant from recharge area, TF-811 and -815 , which were closer to mine, gave high delta values as compared to the other 2 samples. TF-813 coll. $4 \mathrm{mi} \mathrm{S}$ and TF-817 $6 \mathrm{mi} \mathrm{N}$, whereas other samples of series were 25 $\mathrm{mi} \mathrm{NE}$ of mine.

Free $\mathrm{CO}_{2}$ from tube well water. Coll. 1967 and subm. by S. K. Gupta.

\begin{tabular}{|c|c|c|c|c|}
\hline Lab. no. & Field no. & $\begin{array}{l}\text { Well no. or } \\
\text { location }\end{array}$ & $\begin{array}{l}\text { Uncorrected } \\
\mathrm{C}^{14} \text { age }\end{array}$ & $\%$ of Modern \\
\hline $\mathrm{TF}-810$ & NV/SKG-11/9 & Nellikuppam & $\begin{array}{l}1020 \pm 100 \\
\text { A.D. } 930\end{array}$ & $88.18 \pm 1.22 \%$ \\
\hline TF-811 & NV/SKG-4/2 & NP-8 & $\begin{array}{c}370 \pm 95 \\
\text { A.D. } 1580\end{array}$ & $95.58 \pm 1.2 \%$ \\
\hline TF-813 & NV/SKG-8/6 & J.E. Qts. & $\begin{array}{l}3775 \pm 100 \\
1825 \text { B.C. }\end{array}$ & $62.50 \pm 1.2 \%$ \\
\hline TF-815 & NV/SKG-3/1 & SP-18 & $\begin{array}{r}65 \pm 95 \\
\text { A.D. } 1885\end{array}$ & $99.21 \pm 1.2 \%$ \\
\hline TF-816 & NV/SKG-6/4 & Cuddalore & $\begin{array}{l}1790 \pm 95 \\
\text { A.D. } 160\end{array}$ & $80.03 \pm 1.17 \%$ \\
\hline TF-817 & NV/SKG-5/3 & 4th BL & $\begin{array}{l}2895 \pm 95 \\
945 \text { B.C. }\end{array}$ & $69.77 \pm 1.2 \%$ \\
\hline TF-818 & $\mathrm{NV} / \mathrm{SKG}-7 / 5$ & Pondichery & $\begin{array}{l}4945 \pm 115 \\
2995 \text { B.C. }\end{array}$ & $54.05 \pm 1.41 \%$ \\
\hline
\end{tabular}

Date list:

RFFERENCIS

Tata Institute V Agrawal and Kusumgar, 1968

Agrawal, D. P. and Kusumgar, Sheela, 1968, Tata Institute radiocarbon date list V: Radiocarbon, v. 10, p. 131-I43.

Raikes, R. L., 1963, New prehistoric bichrome ware from the plains of Baluchistan (West Pakistan): East and West (Rome), v. 14, p. 56-58.

Sixth internat. conf. radiocarbon and tritium dating, June 7-11, 1965, Pullman, Washington, U.S. Atomic Energy Comm. 